\title{
Glitches detected in southern radio pulsars
}

\author{
Meng $\mathbf{Y u}^{1,2,3 \dagger}$ \\ ${ }^{1}$ School of Physics and State Key Laboratory of Nuclear Physics and Technology, \\ Peking University \\ ${ }^{2}$ CSIRO Astronomy and Space Science \\ ${ }^{3}$ National Astronomical Observatories of China \\ email: vela.yumeng@gmail.com
}

\begin{abstract}
Parkes pulse arrival-time data for 165 radio pulsars spanning from 1990 to 2011 have been searched for period glitches. Forty-six events out of the detected 107 glitches were found to be new contributions to the entire glitch population which currently contains approximately 400 events.
\end{abstract}

Keywords. stars: neutron - pulsars: general

\section{Introduction}

Two types of timing irregularities have been hampering a full interpretation of the rotation of neutron stars: timing noise - a random process seen in phase residuals and glitches - a discontinuous increase in the pulse frequency $\nu$. Glitches are transient events; the most stringent upper-bound known for the timescale of the rising edge of $\nu, 40 \mathrm{~s}$, was obtained by Dodson, McCulloch \& Lewis (2002) in a high time-resolution observing program for the Vela pulsar. The frequency jump caused by a glitch is actually small with the detected maximum relative size of $\Delta \nu_{\mathrm{g}} / \nu \sim 10^{-5}$ (Yuan et al. 2010; Manchester \& Hobbs 2011). After a glitch, the slow-down rate $|\dot{\nu}|$ often partially recovers exponentially, followed by a long-term linear decrease; permanent changes in $\nu$ and its time derivatives are usually left. These widely observed features are used to model glitches.

\section{Observations and Results}

Approximately 200 Galactic pulsars have been being observed regularly from 1990 up until now by the 64-m radio telescope at the Parkes Observatory located in western NSW, Australia. Fig. 1 shows 165 pulsars that are in the sample on the $P-\dot{P}$ diagram. Each pulse time-of-arrival (TOA) for a particular pulsar was obtained every two to four weeks. The data spans for these pulsars range from 5.3 to $20.8 \mathrm{yr}$ with a total of $\sim 1911 \mathrm{yr}$. By carrying out off-line data reduction, 107 glitches were identified in 36 pulsars and 46 events have not previously been published. Moreover, the recovery fraction $Q$ and the timescale $\tau_{\mathrm{d}}$ were measured for 27 exponential decays; post-glitch $\ddot{\nu}$ were also well measured for those pulsars that exhibit long-term linear increase in $\dot{\nu}$ between two adjacent glitches. More detailed results are being published in Yu et al. (2012).

\section{Discussion}

Young pulsars have been thought to be appropriate samples to study neutron-star interiors. It was soon after the discovery of the first glitch that led to the realisation

$\dagger$ Present address: 20A Datun Road, Chaoyang District, Beijing 100012, P. R. China 


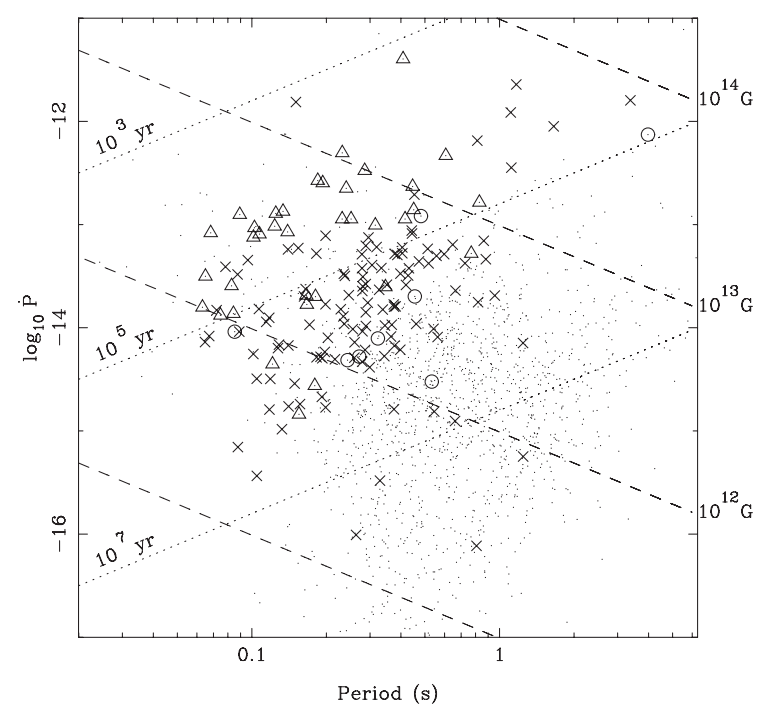

Figure 1. $P-\dot{P}$ diagram showing the 165 pulsars in the sample. The various symbols indicate the pulsars where no glitch has been reported $(\times)$, glitches were detected within our data span $(\triangle)$ and glitches were previously reported before our observations (O). Data for the pulse period and period derivative are from the ATNF Pulsar Catalogue (http://www.atnf.csiro.au/research/pulsar/psrcat/; version 1.43).

that neutron stars are two-component rotators: a rigid-body bulk plus a faster-rotating neutron superfluid (Baym et al. 1969). After twenty-year-observation at Parkes, we now have further found i) the bimodal distribution of the glitch fractional size $\Delta \nu_{\mathrm{g}} / \nu$ has been even clearer (upper panel, Fig. 2), ii) glitches have frequently occurred in the pulsars that have characteristic ages around $10 \mathrm{kyr}$ where, furthermore, large glitches were generally seen, iii) exponential recoveries could be resolved into multiple components corresponding to different timescales and iv) post-glitch $\ddot{\nu}$ values are often positive, are generally larger than the prediction from the magnetic-dipole model and are proportional to the ratio between the slow-down rate and the inter-glitch interval with a proportionality constant $\sim 10^{-3}$. These observations have further probed that neutron stars may suffer the re-configuration of the crustal plate tectonics and/or the substantial release of pinned vortices and, the rotational equilibrium after a glitch established via vortex-drifting may occur in multiple regimes resulting in the observed exponential and linear recoveries.

Never-the-less, the available observations are still not sufficient to entirely interpret the glitch phenomenon. As shown in the lower panel of Fig. 2, the observed fraction $Q$ of the glitch that recovers exponentially has exhibited a bimodal distribution with two peaks locating at approximately 0.01 and 1.0 respectively. For the two-component model, the conservation of angular momentum indicates $Q=I_{\mathrm{s}} / I$, where $I$ is the total moment of inertia of the neutron star and $I_{\mathrm{s}}$ the inertial moment of the superfluid that gradually couples to the effective crust following a glitch. The values of $Q \sim 1$ might have implied that, for some glitches, $I_{\mathrm{S}}$ involved most of the neutron superfluid, though it has been shown that the core superfluid may rigidly couple to the realistic crust in a timescale $\lesssim$ seconds (Alpar, Langer \& Sauls 1984; Alpar \& Sauls 1988). Moreover, a very large $Q \sim 8.7$ was detected in PSR J1846-0258 recently (Livingstone et al. 2010) which is an even more complicated situation. In addition, peculiar features have also been observed. For instance, quasi-periodic oscillation in timing residuals following a glitch has both been seen in PSR B2334+61 (Yuan et al. 2010) and the Vela pulsar where, moreover, 

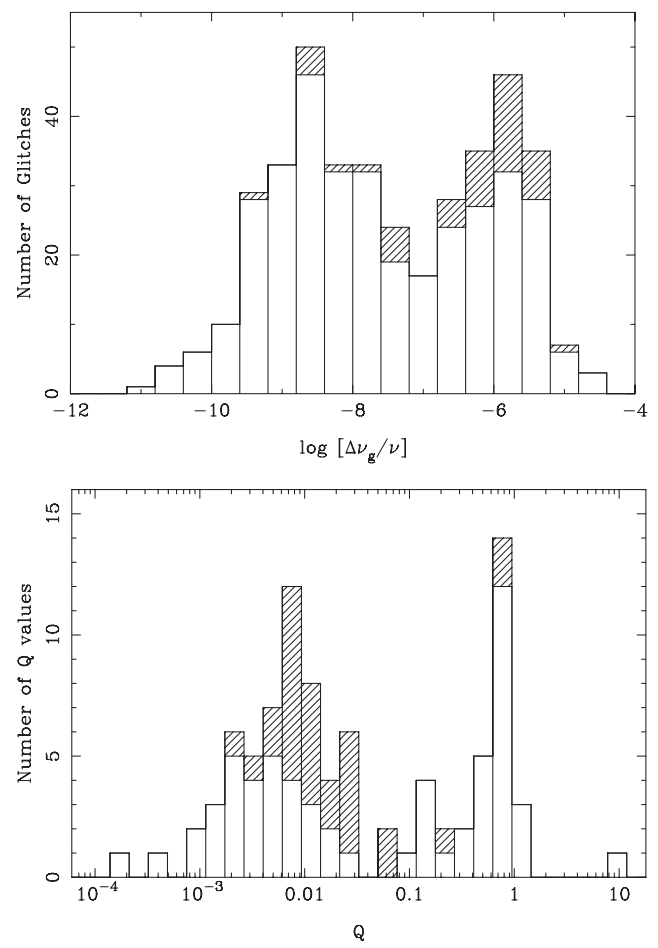

Figure 2. Histograms for the fractional glitch size $\Delta \nu_{\mathrm{g}} / \nu$ (upper panel) and the recovery fraction $Q$ of exponential decays (lower panel). In each plot, the data for the blank bars are from the literature, whereas those for the shaded bars are from $\mathrm{Yu}$ et al. (2012).

excess delays were observed for the pulse arrival times measured at a lower frequency (McCulloch et al. 1990). Further exploration on glitch-related phenomena may require intensive observations perhaps with high precision and high observing cadence.

\section{Acknowledgements}

MY acknowledges the National Basic Research Program of China (2012CB821800) and China Scholarship Council (No. 2009601129) for funding. The Parkes radio telescope is part of the Australia Telescope, which is funded by the Commonwealth of Australia for operation as a National Facility managed by the Commonwealth Scientific and Industrial Research Organisation (CSIRO).

\section{References}

Alpar M. A., Langer S. A., \& Sauls J. A., 1984, ApJ, 282, 533

Alpar M. A. \& Sauls J. A., 1988, ApJ, 327, 723

Baym G., Pethick C., Pines D., \& Ruderman M., 1969, Nature, 224, 872

Dodson R. G., McCulloch P. M., \& Lewis D. R., 2002, ApJ, 564, L85

Livingstone M. A., Kaspi V. M., \& Gavriil F. P., 2010, ApJ, 710, 1710

Manchester R. N. \& Hobbs G., 2011, ApJ, 736, L31

McCulloch P. M., Hamilton P. A., McConnell D., \& King E. A., 1990, Nature, 346, 822

Yu M., Manchester R. N., Hobbs G., Johnston S., Kaspi V. M., Keith M., Lyne A. G., Qiao G.

J., Ravi V., Sarkissian J., Shannon R., \& Xu R. X., MNRAS, submitted

Yuan J. P., Manchester R. N., Wang N., Zhou X., Liu Z. Y., \& Gao Z. F., 2010, ApJ, 719, L111 